\title{
Multi-dimensional Modeling of Massive Binary Interaction in Eta Carinae
}

\author{
J. H. Groh \\ Max-Planck-Institut für Radioastronomie, Auf dem Hügel 69, D-53121 Bonn, Germany \\ email: jgroh@mpifr.de
}

\begin{abstract}
We summarize recent efforts from our group to constrain the nature of both stars in the Eta Carinae binary system and its orbital parameters by studying the influence of the companion star on the spectrum of the primary star. We find that the cavity in the dense wind of the primary star strongly affects multi-wavelength diagnostics such as the ultraviolet spectrum, the optical hydrogen lines, and the shape of the near-infrared continuum region. These diagnostics have been previously interpreted as requiring a latitude-dependent wind generated by a fast-rotating primary star, but the effects of the companion on them provide tenuous evidence that the primary star is a rapid rotator.
\end{abstract}

Keywords. stars: winds, outflows; stars: binaries; stars: emission line

Eta Carinae is one of the most luminous objects in the Galaxy, and its study provides crucial constraints on the evolution and death of the most massive stars. Extensive observational monitoring of Eta Car in the optical and X-rays revealed that Eta Car is a massive binary system consisting of two very massive stars, $\eta_{\mathrm{A}}$ (primary) and $\eta_{\mathrm{B}}$ (secondary), with a total system mass amounting to at least $110 M_{\odot}$ (Hillier et al. 2001). Although most orbital parameters of the system are uncertain, the wealth of multiwavelength observations are consistent with a high eccentricity $(e \sim 0.9)$ and an orbital period of $2022.7 \pm 1.3 \mathrm{~d}$ (Damineli et al. 2008).

To understand and quantify the effects of $\eta_{\mathrm{B}}$ on the wind of $\eta_{\mathrm{A}}$, we developed a twodimensional radiative transfer code to model massive binaries with extended winds. In the case of Eta Car, our models are based on the spherically symmetric models of $\eta_{\mathrm{A}}$ (Hillier et al. 2001), but use the 2-D code of Busche \& Hillier (2005) to study the influence of the low-density cavity, and dense interaction-region walls, on the spectrum. Further details are given in Groh et al. (2010) and Groh et al. (2011, in prep.).

We approximate the cavity as a conical surface with half-opening angle $\alpha$ and interior density 0.0016 times lower than that of the spherical wind model of $\eta_{\mathrm{A}}$. We include cone walls of angular thickness $\delta \alpha$ and, assuming mass conservation, a density contrast in the wall of $f_{\alpha}=[1-\cos (\alpha)] /[\sin (\alpha) \delta \alpha]$ times higher than the wind density of the spherical model of $\eta_{\mathrm{A}}$ at a given radius. The conical shape is justified since the observations we model here were taken at orbital phases sufficiently before periastron, when such a cavity has an approximately 2-D axisymmetric conical form (Okazaki et al. 2008). Based on the expected location of the cone apex during these phases (Okazaki et al. 2008), we place the cavity at a distance $d_{\text {apex }}$ from the primary star. We assume that the material inside the cavity and along the walls has the same ionization structure as the wind of $\eta_{\mathrm{A}}$. Thus, at this point we explicitly neglect the ionization changes that might occur in the wind-wind interacting region. We also do not account for the ionizing flux of $\eta_{\mathrm{B}}$. Despite these limitations, our implementation should be adequate for understanding how the line profiles of $\eta_{\mathrm{A}}$ are modified by the carving of its wind by $\eta_{\mathrm{B}}$. 


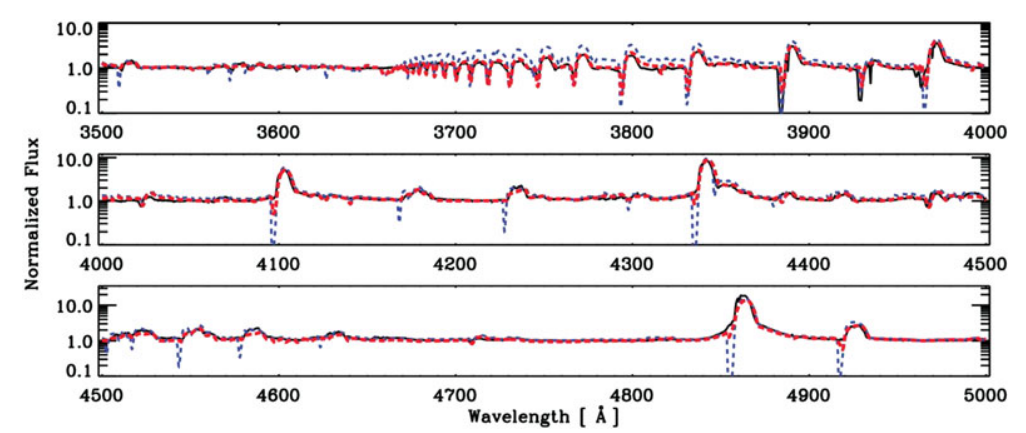

Figure 1. Optical spectrum of $\eta_{\mathrm{A}}$ observed with HST/STIS at $\phi \sim 0.6$ (solid black line) compared to the spherically-symmetric CMFGEN model (blue dashed line) and with our 2-D model (red dashed line). See text for the model parameters.

Here, we illustrate how the optical spectrum is affected by the cavity created by the companion star in the wind of the primary. Assuming a single-star scenario with a spherically-symmetric stellar wind, Hillier et al. (2001) obtained a reasonable fit to the observed HST/STIS optical spectrum obtained right after periastron, at orbital phase $\phi \sim 0.04$. However, the spherically-symmetric CMFGEN model overestimates the amount of P-Cygni absorption. The comparison is even worse as one moves toward apastron $(\phi \sim 0.5)$, when the observations show little or no P-Cygni absorption components in $\mathrm{H}$ and Fe II lines (Fig. 1). This has been interpreted as if $\eta_{\mathrm{A}}$ would be a rapid rotator and its wind, latitude dependent (Hillier et al. 2001, Smith et al. 2003).

Using our 2-D radiative transfer model, which takes into account the cavity in the wind of $\eta_{\mathrm{A}}$ caused by $\eta_{\mathrm{B}}$, we computed the synthetic optical spectrum of Eta Car at $\phi \sim 0.6$ (Fig. 1). We assumed the same parameters described above for $\eta_{\mathrm{A}}$ (Hillier et al. 2001) and a standard geometry of the cavity at apastron as predicted by 3 -D hydrodynamical simulations (Okazaki et al. 2008): $d_{\text {apex }}=25 \mathrm{AU}, \alpha=54^{\circ}, \delta \alpha=3^{\circ}$ (i.e., $f_{\alpha}=9.7$ ), $b=0.0016$. For a viewing angle of $i=41^{\circ}$ and longitude of periastron of $\omega=270^{\circ}$, the 2-D model produces a much better fit to the P-Cygni absorption line profiles of $\mathrm{H}$ and Fe II lines than the 1-D CMFGEN model, while still fitting the emission line profiles. The improved fit to the P-Cygni absorption line profiles yielded by the 2-D model is due to the cavity in the wind of $\eta_{\mathrm{A}}$, which reduces the $\mathrm{H}$ and Fe II optical depths in the line-of-sight to $\eta_{\mathrm{A}}$ when the observer looks down the cavity.

We find that $\eta_{\mathrm{B}}$ significantly affects the $\mathrm{H}$ line profiles, which is one of the main diagnostics of rapid rotation in $\eta_{\mathrm{A}}$. Therefore, an intrinsic latitude-dependent wind generated by fast rotation of $\eta_{\mathrm{A}}$ may not be the only explanation for existing observations, but this does not mean that $\eta_{\mathrm{A}}$ is not a rapid rotator. As the presence of the cavity and walls affects also the available interferometric observables (Groh et al. 2010), which were the other diagnostics supporting fast rotation, tenuous evidence supports a fast-rotating primary star.

\section{References}

Busche, J. R. \& Hillier, D. J. 2005, AJ, 129, 454

Damineli, A., Hillier, D. J., Corcoran, M. F., et al., 2008, MNRAS, 384, 1649

Groh, J. H., Madura, T. I., Owocki, S. P., Hillier, D. J., \& Weigelt, G. 2010, ApJ (Letters), 716, L223

Hillier, D. J., Davidson, K., Ishibashi, K., \& Gull, T. 2001, ApJ, 553, 837

Okazaki, A. T., Owocki, S. P., Russell, C. M. P., \& Corcoran, M. F. 2008, MNRAS, 388, L39

Smith, N., Davidson, K., Gull, T. R., Ishibashi, K., \& Hillier, D. J. 2003, ApJ, 586, 432 